\title{
A NOTE ON GEOMETRICAL FACTORIAL SERIES
}

\author{
BY G. W. STARCHER
}

1. Introduction. This note is concerned with series of the form

$$
c_{0}+\sum_{\nu=1}^{\infty} \frac{c_{\nu}}{(x-q)\left(x-q^{2}\right) \cdots\left(x-q^{\nu}\right)},
$$

where $q$ is such that $|q|<1$. Series of this form are called geometrical factorial series. By comparing (1) with the descending power series

$$
c_{0}+\sum_{\nu=1}^{\infty} c_{\nu} x^{-\nu}
$$

it is shown that they have precisely the same points of convergence, and hence a number of fundamental properties of (1) follow from the known properties of the descending power series. In $\$ 3$ a fundamental theorem concerning the representation of analytic functions by means of series of the form (1) is proved. In $\$ 4$ precise formulas for the multiplication of two such series are obtained. The points $x=q^{i},(i=1,2,3, \cdots)$, will be called exceptional points for the series (1), and we agree to exclude such points from consideration. If we replace $x$ by $x^{-1}$, the series (1) becomes

$$
c_{0}+\sum_{\nu=1}^{\infty} \frac{c_{\nu} x^{\nu}}{(1-q x)\left(1-q^{2} x\right) \cdots\left(1-q^{\nu} x\right)},
$$

which, if it converges at all, converges for values of $x$ in the neighborhood of the origin and defines an analytic function in such a region. Such series might be compared to the ascending power series. This discussion is presented with reference to the series (1) only because of convenience and simplicity of formulas.

2. Comparison of (1) and (2). Suppose $x$ to be a point of convergence of (2). Let us write

$$
u_{\nu}=c_{\nu} x^{-\nu}, \quad v_{\nu}=\frac{x^{\nu}}{(x-q)\left(x-q^{2}\right) \cdots\left(x-q^{\nu}\right)} .
$$


Then

$$
\begin{aligned}
\left|v_{\nu+1}-v_{\nu}\right| & =\left|\left\{\frac{x^{\nu}}{(x-q)\left(x-q^{2}\right) \cdots\left(x-q^{\nu}\right)}\right\}\left\{\frac{x}{x-q^{\nu+1}}-1\right\}\right| \\
& =\left|\frac{q^{\nu+1} x^{\nu}}{(x-q)\left(x-q^{2}\right) \cdots\left(x-q^{\nu+1}\right)}\right| .
\end{aligned}
$$

By the ratio test for convergence we can show that the series $\sum_{\nu=1}^{\infty}\left|v_{\nu+1}-v_{\nu}\right|$ converges for every $x$ not already excluded. By a known theorem* we have the result that

$$
\sum_{\nu=0}^{\infty} u_{\nu} v_{\nu}=c_{0}+\sum_{\nu=1}^{\infty} \frac{c_{\nu}}{(x-q)\left(x-q^{2}\right) \cdots\left(x-q^{\nu}\right)}
$$

converges for all values of $x$ for which (2) converges.

Conversely, let $x$ be a point of convergence of the series (1). Let

$$
\begin{aligned}
& u_{\nu}=\frac{c_{\nu}}{(x-q)\left(x-q^{2}\right) \cdots\left(x-q^{\nu}\right)}, \\
& v_{\nu}=\frac{(x-q)\left(x-q^{2}\right) \cdots\left(x-q^{\nu}\right)}{x^{\nu}} .
\end{aligned}
$$

It is easy to show by the ratio test that the series $\sum_{\nu=1}^{\infty}\left|v_{\nu+1}-v_{\nu}\right|$ converges for every $x$, and hence the series

$$
\sum_{\nu=1}^{\infty} u_{\nu} v_{\nu}=c_{0}+\sum_{\nu=1}^{\infty} c_{\nu} x^{-\nu}
$$

converges at the points of convergence of (1). It follows that the theorems concerning the region of convergence and expressions for the radius of convergence known for the power series (2) also apply to the series (1). By means of the second lemma in the paper already cited we can establish the corresponding theorems for uniform convergence when we exclude the exceptional points by enclosing them in arbitrarily small circles.

3. Representation of Functions by Series of the Form (1). We shall establish the following theorem.

TheOREM. Let $f(x)$ be analytic outside the circle $S$ of radius $R$ about the origin. Then $f(x)$ can be represented uniquely by a series of the form

* See, for example, Carmichael, Transactions of this Society, vol. 17 (1916), p. 211. 
where

$$
c_{0}+\sum_{\nu=1}^{\infty} \frac{c_{\nu}}{(x-q)\left(x-q^{2}\right) \cdots\left(x-q^{\nu}\right)}, \quad|q|<1,
$$

$$
c_{k}=\frac{1}{2 \pi i} \int_{C}(z-q)\left(z-q^{2}\right) \cdots\left(z-q^{k-1}\right) f_{0}(z) d z, \quad k>0,
$$

$f_{0}(z)$ being written for $f(z)-c_{0}$. The contour $C$ is understood to be any contour about the origin lying entirely outside $S$, such that the point $x$ lies outside $C$, and $C$ does not pass through any exceptional points.

Let $f(\infty)=c_{0}$. Consider the function $f_{0}(x)=f(x)-c_{0}$. By the Cauchy integral theorem we have*

$$
f_{0}(x)=-\frac{1}{2 \pi i} \int_{C} \frac{f_{0}(z) d z}{z-x}+\frac{1}{2 \pi i} \int_{C^{\prime}} \frac{f_{0}(z) d z}{z-x},
$$

where $C$ and $C^{\prime}$ are circles lying outside $S$ and are such that the point $x$ lies inside $C^{\prime}$ but is outside $C$. By letting the radius of $C^{\prime}$ increase indefinitely we can show that the second integral contributes zero, since $\lim _{z \rightarrow \infty} f_{0}(z)=0$, and we have finally

$$
f_{0}(x)=\frac{1}{2 \pi i} \int_{c} \frac{f_{0}(z) d z}{x-z} .
$$

We can easily verify the following algebraic identity:

$$
\frac{1}{x-z}=\frac{1}{x-q^{s}}+\frac{z-q^{s}}{x-q^{s}} \cdot \frac{1}{x-z} .
$$

If in this expression we first take $s=1$, then replace $1 /(x-z)$ in the second member by its value above when $s=2$, again when $s=3$, and continue $k$ times, we have the algebraic identity

$$
\begin{gathered}
\frac{1}{x-z}=\frac{1}{x-q}+\frac{z-q}{x-q} \cdot \frac{1}{x-z} \\
=\frac{1}{x-q}+\frac{z-q}{(x-q)\left(x-q^{2}\right)}+\frac{(z-q)\left(z-q^{2}\right)}{(x-q)\left(x-q^{2}\right)} \cdot \frac{1}{x-z} \\
=\frac{1}{x-q}+\sum_{j=1}^{k-1} \frac{(z-q)\left(z-q^{2}\right) \cdots\left(z-q^{j}\right)}{(x-q)\left(x-q^{2}\right) \cdots\left(x-q^{j+1}\right)} \\
+\frac{(z-q) \cdots\left(z-q^{k}\right)}{(x-q) \cdots\left(x-q^{k}\right)} \cdot \frac{1}{x-z} .
\end{gathered}
$$

* See Whittaker and Watson, Modern Analysis, 4th ed., \$5.21. 
Then by (3) and (5) we have

$$
\begin{aligned}
f_{0}(x)= & \frac{1}{2 \pi i} \int_{C} \frac{f_{0}(z) d z}{x-z} \\
= & \sum_{j=0}^{k-1} \frac{1}{2 \pi i} \int_{C} \frac{(z-q)\left(z-q^{2}\right) \cdots\left(z-q^{j}\right) f_{0}(z)}{(x-q)\left(x-q^{2}\right) \cdots\left(x-q^{j+1}\right)} d z \\
& +\frac{1}{2 \pi i} \int_{C} \frac{(z-q)\left(z-q^{2}\right) \cdots\left(z-q^{k}\right) f_{0}(z)}{(x-q)\left(x-q^{2}\right) \cdots\left(x-q^{k}\right)(x-z)} d z .
\end{aligned}
$$

Since $z$ lies on $C$ we have the following inequality, $|z| /|x| \leqq \rho<1$. We denote by $R_{k}$ the last term in (6). For every positive number $\epsilon$ there exists an integer $k_{0}$ such that for $k>k_{0}$ we can write

Then we have

$$
\left|\frac{z-q^{k}}{x-q^{k}}\right|<\rho+\epsilon \text {. }
$$

$$
\begin{gathered}
\left|\frac{1}{2 \pi i} \int_{C} \frac{(z-q)\left(z-q^{2}\right) \cdots\left(z-q^{k_{0}}\right)\left(z-q^{k_{0}+1}\right) \cdots\left(z-q^{k}\right) f_{0}(z)}{(x-q)\left(x-q^{2}\right) \cdots\left(x-q^{k_{0}}\right)\left(x-q^{k_{0}+1}\right) \cdots\left(x-q^{k}\right)(x-z)} d z\right| \\
\leqq \frac{M}{2 \pi}\left|\int_{C}(\rho+\epsilon)^{k-k_{0}} d z\right|=M(\rho+\epsilon)^{k-k_{0}}, \quad k>k_{0} .
\end{gathered}
$$

When $\epsilon$ is sufficiently small, $\rho+\epsilon<1$ and $\lim _{k \rightarrow \infty} M(\rho+\epsilon)^{k-k_{0}}=0$. That is, $\lim _{k \rightarrow \infty} R_{k}=0$. From (6) we have

(7) $f_{0}(x)=\frac{1}{2 \pi i} \int_{C} \frac{f_{0}(z) d z}{x-q}$

$$
+\sum_{\nu=2}^{\infty} \frac{1}{2 \pi i} \int_{C} \frac{(z-q)\left(z-q^{2}\right) \cdots\left(z-q^{\nu-1}\right) f_{0}(z)}{(x-q)\left(x-q^{2}\right) \cdots\left(x-q^{\nu}\right)} d z .
$$

This series converges and represents the analytic function $f_{0}(x)$ outside the circle $S$. Then we have

(8) $f(x)=c_{0}+f_{0}(x)=c_{0}+\sum_{\nu=1}^{\infty} \frac{c_{\nu}}{(x-q)\left(x-q^{2}\right) \cdots\left(x-q^{\nu}\right)}$,

where

$$
c_{k}=\frac{1}{2 \pi i} \int_{C}(z-q)\left(z-q^{2}\right) \cdots\left(z-q^{k-1}\right) f_{0}(z) d z, \quad k>0,
$$

as the theorem states. 
Finally, there can be but one such expansion. For suppose we also had

$$
f(x)=d_{0}+\sum_{\nu=1}^{\infty} \frac{d_{\nu}}{(x-q)\left(x-q^{2}\right) \cdots\left(x-q^{\nu}\right)} .
$$

Subtracting (9) from (8) member by member gives

$$
0=c_{0}-d_{0}+\sum_{\nu=1}^{\infty} \frac{c_{\nu}-d_{\nu}}{(x-q)\left(x-q^{2}\right) \cdots\left(x-q^{\nu}\right)} .
$$

Considering the limit approached by this expression as $x$ becomes infinite we have $c_{0}-d_{0}=0$, or $c_{0}=d_{0}$. Then

$$
0=\sum_{\nu=1}^{\infty} \frac{c_{\nu}-d_{\nu}}{(x-q)\left(x-q^{2}\right) \cdots\left(x-q^{\nu}\right)} .
$$

Multiplying through by $x-q$ gives

$$
0=c_{1}-d_{1}+\sum_{\nu=2}^{\infty} \frac{c_{\nu}-d_{\nu}}{\left(x-q^{2}\right)\left(x-q^{3}\right) \cdots\left(x-q^{\nu}\right)} .
$$

Again letting $x$ become infinite we have $c_{1}-d_{1}=0$, or $c_{1}=d_{1}$. Continuing this process we have $c_{i}=d_{i}, i=1,2,3, \cdots$, and the expansion (8) is unique.

4. Operations with Series of the Form (1). Clearly the addition of two series of the form (1) presents no difficulty. That the function represented by the product of two series of the form (1) can be expressed in the same form follows from the properties of the series given in the preceding sections of this paper. We are here concerned with obtaining the precise coefficients in such a product.

In (5) let us write $q$ for $z, q^{\nu+1}$ for $q$, then again apply (5) to the second member of the identity after writing $q^{\nu+2}$ for $q$, and continue $k$ times. If we denote the last term in the second member by $L_{k}$, then $\lim _{k \rightarrow \infty} L_{k}=0$ and we can write

$$
\begin{aligned}
\frac{1}{x-q}=\frac{1}{x-q^{\nu+1}} & +\frac{q\left(1-q^{\nu}\right)}{\left(x-q^{\nu+1}\right)\left(x-q^{\nu+2}\right)} \\
& +\frac{q^{2}\left(1-q^{\nu}\right)\left(1-q^{\nu+1}\right)}{\left(x-q^{\nu+1}\right)\left(x-q^{\nu+2}\right)\left(x-q^{\nu+3}\right)}+\cdots .
\end{aligned}
$$


Then we have

$$
\text { (10) } \begin{gathered}
\frac{c_{\nu}}{(x-q) \cdots\left(x-q^{\nu}\right)}=\frac{c_{\nu}}{\left(x-q^{2}\right)\left(x-q^{3}\right) \cdots\left(x-q^{\nu+1}\right)} \\
+\frac{c_{\nu} q\left(1-q^{\nu}\right)}{\left(x-q^{2}\right)\left(x-q^{3}\right) \cdots\left(x-q^{\nu+2}\right)}+\cdots .
\end{gathered}
$$

The latter series converges absolutely and uniformly for all values of $x$ such that $|x| \geqq r>|q|$. Let $r_{1}$ be the radius of absolute convergence of (1). Substituting from (10) into (1) we have a double series which we denote by $c_{0}+\sum_{m, n=1}^{\infty} a_{m n}$. Let $b_{m n}=c_{m} q^{n-1} / x^{m+n-1}$. The series $\sum_{m, n=1}^{\infty} b_{m n}$ is the product of the two series $\sum_{\nu=1}^{\infty} c_{\nu} / x^{\nu}$ and $\sum_{\nu=1}^{\infty} q^{\nu-1} / x^{\nu}$, each of which converges absolutely if $|x|>R$, where $R$ is the larger of the two numbers $r$ and $r_{1}$. By a known theorem* $\sum_{m, n=1}^{\infty} b_{m n}$ converges absolutely in the same region. Since $\prod_{\nu=1}^{\infty}\left(1-q^{\nu}\right)$ and $\prod_{\nu=1}^{\infty}\left(1-q^{\nu+1} / x\right)$, $|x|>R$, represent convergent infinite products we can easily show that the ratio $\left|a_{m n} / b_{m n}\right|<M$, where $M$ is finite. Hence $\sum_{m, n=1}^{\infty}\left|a_{m n}\right|<M \sum_{m, n=1}^{\infty} b_{m n}$ and the double series $\sum_{m, n=1}^{\infty} a_{m n}$ converges absolutely at the points of absolute convergence of $\sum_{m, n=1}^{\infty} b_{m n}$, that is, outside the circle of radius $R$. By a well known theorem, $\uparrow$ the double series obtained above gives the same sum for any mode of summation. Summing by diagonals gives

(11) $c_{0}+\sum_{\nu=1}^{\infty} \frac{S_{\nu}^{(1)}}{\left(x-q^{2}\right)\left(x-q^{3}\right) \cdots\left(x-q^{\nu+1}\right)}, \quad|x|>R$, where

$$
S_{\nu}^{(1)}=\sum_{i=1}^{\nu} q^{\nu-i}\left(1-q^{i}\right)\left(1-q^{i+1}\right) \cdots\left(1-q^{\nu-1}\right) c_{i} .
$$

In the same way we can show that the series (11) can be transformed into the series

$$
c_{0}+\sum_{i=1}^{\infty} \frac{S_{\nu}^{(2)}}{\left(x-q^{3}\right)\left(x-q^{4}\right) \cdots\left(x-q^{\nu+2}\right)},
$$

where

$$
S_{\nu}^{(2)}=\sum_{i=1}^{\nu} q^{2(\nu-i)}\left(1-q^{i}\right)\left(1-q^{i+1}\right) \cdots\left(1-q^{\nu-1}\right) S_{i}^{(1)}
$$

* See Bromwich, Theory of Infinite Series, p. 90.

$\dagger$ Bromwich, Theory of Infinite Series, $\$ 31$. 
and finally into

where

$$
c_{0}+\sum_{\nu=1}^{\infty} \frac{S_{\nu}^{(k+1)}}{\left(x-q^{k+2}\right)\left(x-q^{k+3}\right) \cdots\left(x-q^{k+\nu+1}\right)},
$$

$$
S_{\nu}^{(k+1)}=\sum_{i=1}^{\nu} q^{(k+1)(\nu-i)}\left(1-q^{i}\right)\left(1-q^{i+1}\right) \cdots\left(1-q^{\nu-1}\right) S_{i}^{(k)} .
$$

Using this formula repeatedly we have

$$
\begin{gathered}
S_{\nu}^{(k)}=\sum q^{k(\nu-i)}\left(1-q^{i}\right)\left(1-q^{i+1}\right) \cdots\left(1-q^{\nu-1}\right) q^{(k-1)(\nu-j)}\left(1-q^{j}\right) \\
\cdots\left(1-q^{\nu-1}\right) \cdots q^{\nu-s}\left(1-q^{s}\right)\left(1-q^{s+1}\right) \cdots\left(1-q^{\nu-1}\right) c_{s},
\end{gathered}
$$

where the summation is understood to be taken for all possible integral values of $\nu, i, j, \cdots, s$, such that

$$
1 \leqq s \leqq \cdots \leqq j \leqq i \leqq \nu .
$$

\section{LEMMA.}

$$
\begin{aligned}
& \left(1-q^{\alpha+1}\right)\left(1-q^{\alpha+2}\right) \cdots\left(1-q^{\alpha+k-1}\right) \\
& \quad+q^{k}\left(1-q^{\alpha}\right)\left(1-q^{\alpha+1}\right) \cdots\left(1-q^{\alpha+k-2}\right) \\
& \quad+q^{2 k}\left(1-q^{\alpha-1}\right)\left(1-q^{\alpha}\right) \cdots\left(1-q^{\alpha+k-3}\right)+\cdots \\
& \quad+q^{(\alpha-1) k}\left(1-q^{2}\right)\left(1-q^{3}\right) \cdots\left(1-q^{k}\right) \\
& \quad+q^{\alpha k}(1-q)\left(1-q^{2}\right) \cdots\left(1-q^{k-1}\right) \\
& \quad=\left(1-q^{\alpha+1}\right)\left(1-q^{\alpha+2}\right) \cdots\left(1-q^{\alpha+k}\right) /\left(1-q^{k}\right) .
\end{aligned}
$$

By combining terms in the first member two at a time, at each step taking the last two terms and continuing backward until all the terms are combined, we have the second member.

By simple induction we can establish the formulas

$$
\begin{aligned}
& S_{\nu}^{(2)}=\sum_{i=0}^{\nu-1} \frac{q^{i}\left(1-q^{i+1}\right)\left(1-q^{\nu-i}\right)\left(1-q^{\nu-i+1}\right) \cdots\left(1-q^{\nu-1}\right) c_{\nu-i}}{1-q}, \\
& S_{\nu}^{(3)}=\sum_{i=0}^{\nu-1} \frac{q^{i}\left(1-q^{i+1}\right)\left(1-q^{i+2}\right)\left(1-q^{\nu-i}\right)\left(1-q^{\nu-i+1}\right) \cdots\left(1-q^{\nu-1}\right) c_{\nu-i}}{(1-q)\left(1-q^{2}\right)},
\end{aligned}
$$

and finally, by using the lemma above, we have by induction

$$
S_{\nu}^{(k)}=\sum_{i=0}^{\nu-1} \frac{q^{i}\left(1-q^{i+1}\right) \cdots\left(1-q^{i+k-1}\right)\left(1-q^{\nu-i}\right) \cdots\left(1-q^{\nu-1}\right) c_{\nu-i}}{(1-q)\left(1-q^{2}\right) \cdots\left(1-q^{k-1}\right)} .
$$


Consider the product

$$
\begin{aligned}
{\left[c_{0}\right.} & \left.+\sum_{\nu=1}^{\infty} \frac{c_{\nu}}{(x-q) \cdots\left(x-q^{\nu}\right)}\right]\left[d_{0}+\sum_{\nu=1}^{\infty} \frac{d_{\nu}}{(x-q) \cdots\left(x-q^{\nu}\right)}\right] \\
= & c_{0} d_{0}+\sum_{\nu=1}^{\infty} \frac{c_{\nu} d_{0}+c_{0} d_{\nu}}{(x-q) \cdots\left(x-q^{\nu}\right)} \\
& +\left[\sum_{\nu=1}^{\infty} \frac{c_{\nu}}{(x-q) \cdots\left(x-q^{\nu}\right)}\right]\left[\sum_{\nu=1}^{\infty} \frac{d_{\nu}}{(x-q) \cdots\left(x-q^{\nu}\right)}\right] .
\end{aligned}
$$

Since we have

$$
\begin{aligned}
& \sum_{\nu=1}^{\infty} \frac{c_{\nu}}{(x-q) \cdots\left(x-q^{\nu}\right)} \\
= & \sum_{\nu=1}^{\infty} \frac{S_{\nu}^{(k)}}{\left(x-q^{k+1}\right)\left(x-q^{k+2}\right) \cdots\left(x-q^{k+\nu}\right)},
\end{aligned}
$$

the above product becomes

$$
c_{0} d_{0}+\sum_{\nu=1}^{\infty} \frac{A_{\nu}}{(x-q)\left(x-q^{2}\right) \cdots\left(x-q^{\nu}\right)},
$$

where*

$$
A_{\nu}=d_{0} c_{\nu}+d_{\nu} c_{0}+\sum_{i=1}^{\nu-1} S_{i}^{(\nu-i)}(c) d_{\nu-i}
$$

Within the region of uniform convergence of (1) the terms are all analytic functions and hence by a well known theorem $\dagger$ the series (1) can be differentiated term by term. If in (5) we write $q^{t}$ for $z, q^{\nu+1}$ for $q$, and denote the last term in the second member by $L_{k}{ }^{\prime}$, then $\lim _{k \rightarrow \infty} L_{k}{ }^{\prime}=0$ and we can write

$$
\begin{array}{r}
\frac{1}{x-q^{t}}=\sum_{i=1}^{\infty} \frac{q^{(i-1) t}\left(1-q^{\nu-t+1}\right)\left(1-q^{\nu-t+2}\right) \cdots\left(1-q^{\nu-t+i-1}\right)}{\left(x-q^{\nu+1}\right)\left(x-q^{\nu+2}\right) \cdots\left(x-q^{\nu+i}\right)}, \\
x \geqq r>|q| .
\end{array}
$$

Using identities of this form to transform the series in the second member below into a geometrical factorial series, we have

* $S_{i}^{(k)}(c)$ is written to indicate that the $S$ 's are formed with the $c$ 's. It will be observed that $A_{\nu}$ is symmetric in the $c$ 's and $d$ 's.

$\dagger$ See for example Townsend, Functions of a Complex Variable, p. 224. 


$$
\begin{aligned}
& \frac{d}{d x}\left\{c_{0}+\sum_{\nu=1}^{\infty} \frac{c_{\nu}}{(x-q)\left(x-q^{2}\right) \cdots\left(x-q^{\nu}\right)}\right\} \\
& =-\sum_{\nu=1}^{\infty} \frac{c_{\nu}}{(x-q)\left(x-q^{2}\right) \cdots\left(x-q^{\nu}\right)}\left\{\frac{1}{x-q}+\frac{1}{x-q^{2}}+\cdots+\frac{1}{x-q^{\nu}}\right\} \\
& =-\sum_{\nu=1}^{\infty} \sum_{j=1}^{\infty} \frac{C_{\nu}{ }^{(j)}}{(x-q)\left(x-q^{2}\right) \cdots\left(x-q^{\nu+j}\right)} \\
& =\sum_{\nu=1}^{\infty} \frac{D_{\nu}}{(x-q)\left(x-q^{2}\right) \cdots\left(x-q^{\nu}\right)},|x| \geqq R,
\end{aligned}
$$

where*

$$
C_{\nu}^{(k)}=\sum_{i=0}^{\nu-1} \prod_{j=0}^{k-2} q^{i+1}\left(1-q^{\nu-i+j}\right) c_{\nu}, \quad D_{\nu}=-\sum_{j=1}^{\nu-1} C_{\nu-j}^{(j)},
$$

and where $R$ is defined above.

Again we can take the indefinite integral of the series (1) within the region of uniform convergence and obtain

$$
\begin{aligned}
& \int\left\{c_{0}+\sum_{\nu=1}^{\infty} \frac{c_{\nu}}{(x-q)\left(x-q^{2}\right) \cdots\left(x-q^{\nu}\right)}\right\} \\
& =a_{0}+a_{1}{ }^{\prime} x+a_{0}{ }^{\prime \prime} \log (x-q) \\
& +\sum_{\nu=1}^{\infty} \frac{a_{\nu}}{(x-q)\left(x-q^{2}\right) \cdots\left(x-q^{\nu}\right)},
\end{aligned}
$$

where $a_{0}$ is the constant of integration,

$$
a_{0}^{\prime}=c_{0}, \quad a_{0}^{\prime \prime}=c_{1}, \quad a_{1}=-c_{2},
$$

and in general $a_{\nu}$ is given by the relation $a_{\nu}=-\frac{1}{\nu}\left\{c_{\nu+1}+\sum_{t=1}^{\nu} \sum_{i=1}^{\nu-t} \prod_{j=1}^{t} q^{i t}\left(1-q^{\nu-t-i+j}\right) a_{\nu-t}\right\}, \nu>1$.

OHIO UNIVERSity

* We interpret the notation so that $C_{\nu}^{\prime}=\nu c_{\nu}$ and $D_{1}=0$. 